\title{
The Effects of Antral Preservation and Antral Resection on Body Composition, Glycemic Control and Bone Mineral Density Following Vertical Sleeve Gastrectomy in C57BL/6] Mice with Obesity and Type 2 Diabetes
}

\author{
Xiaoyang $\mathrm{Qi}^{1,2}$, Xugang Li ${ }^{2}$, Yuwen Jiao', Shuai Chen $\mathbb{D}^{\prime}$, Peng Song', Zhifen Qian', Liming Tang' \\ 'Department of Gastrointestinal Surgery, The Affiliated Changzhou No. 2 People's Hospital of Nanjing Medical University, Changzhou, Jiangsu \\ Province, People's Republic of China; ${ }^{2}$ Department of Orthopaedics, Nanjing Drum Tower Hospital, The Affiliated Hospital of Nanjing University \\ Medical School, Nanjing, Jiangsu Province, People's Republic of China
}

Correspondence: Liming Tang, Department of Gastrointestinal Surgery, The Affiliated Changzhou No. 2 People's Hospital of Nanjing Medical University, No. 68, Gehu Middle Road, Changzhou, 213000, Jiangsu, People's Republic of China, Tel +86-I3506I I2690, Email drtangliming@I63.com

\begin{abstract}
Purpose: Sleeve gastrectomy (SG) is the most currently popular operation for obesity and related metabolic disorders. The aim of this study was to compare the effect of antrum preservation SG (AP-SG) and antrum resection SG (AR-SG) on the body composition, glycemic control and bone mineral density (BMD) in mice.

Methods: Sham, AP-SG and AR-SG operation were performed on obese and T2D C57BL/6J mice (8 in each group). Body weight, food intake, and fasting glucose (FG) levels were measured at the $0,2,4,6$ and 8 weeks post-operatively. Oral glucose tolerance test (OGTT) was performed preoperatively and at the eighth postoperative week. The body fat content and total body BMD were evaluated by dual-energy x-ray absorptiometry. After being euthanized, the femurs were harvested and analyzed by micro-CT.

Results: The improvements in body weight, food intake, FG, glycemic control and body fat were statistically significant following AP-SG and AR-SG. Both AP-SG and AR-SG groups decreased total body BMD and regional BMD in the distal femur compared to the sham group. No significant difference of FG was observed in AP-SG and AR-SG group postoperatively, but AR-SG showed significantly superior OGTT glucose AUC than AP-SG. Except for a lower BMD, AR-SG achieved superior outcomes in body fat and glycemic control than AP-SG.

Conclusion: Antrum resection SG shows a lower percentage of body fat and better glycemic control than antrum preservation SG. However, antrum resection SG has a higher risk of having a lower bone mass. Further human clinical trials are needed to confirm this finding.
\end{abstract}

Keywords: sleeve gastrectomy, antrum preservation, antrum resection, body fat, bone mineral density

\section{Introduction}

Bariatric surgery is the most effective treatment for morbid obesity. It improves obesity-related metabolic disorders, the weight-related quality of life, and decreases the overall mortality. ${ }^{1,2}$ Nowadays, sleeve gastrectomy (SG) has become the most popular bariatric procedure that has been recognized for its effective clinical results. ${ }^{3-5}$ Current evidence suggests that in addition to food intake restriction, the accelerated gastric emptying and neurohormonal factors after SG may also be involved in the mechanisms of weight loss. ${ }^{6,7}$

Despite its popularity, SG is not well standardized, and there is still controversy concerning the antrum preservation or resection among the surgeons. The most conservative surgeons prefer to begin the resection at 5-7 $\mathrm{cm}$ from the 
pylorus. Other surgeons performed the radical resection of the antrum with 2-2.5 $\mathrm{cm}$ from the pylorus. Supporters of antrum resection SG (AR-SG) believe that a radical antrum resection causes more restriction leading to maximum weight loss. ${ }^{8}$ Opponents argue that antrum preservation SG (AP-SG) can preserve the contractile function and physiological gastric emptying, in order to avoid increased intragastric pressure and reduce the incidence of postoperative complications. ${ }^{9}$

To date, several studies have evaluated the effect of AP-SG and AR-SG on weight loss outcome. Obeidat et al found that radical antrum resection safely enhanced the restrictive effect and achieved greater and better weight loss compared to antrum preservation. ${ }^{10}$ In the meta-analysis of McGlone et al, it concludes that AR-SG is associated with better medium-term weight loss compared to AP-SG without increased risk of surgical complications. ${ }^{11}$ However, some scholars reported that there was no difference between AP-SG and AR-SG regarding excess weight loss percentage. ${ }^{12-14}$ In addition, several studies have focused on the effect of AP-SG and AR-SG on glycemic control, showing conflicting results. ${ }^{12,15}$ More evidence is required on the matter of the antrum size and its relation to weight loss and glycemic control.

Fracture risk seems to be increased in patients undergoing bariatric surgery. ${ }^{16}$ Bone loss has been reported as early as 6 months after SG. ${ }^{17}$ Misra and Nogués et al found that SG had a significant bone loss in the femoral neck, hip and spine over 12-month follow-up. ${ }^{18,19}$ Progressive skeletal unloading, calcium and vitamin D deficiency caused by malabsorption have been reported to be responsible for bone loss and the development of osteoporosis. ${ }^{20}$ However, there is a lack of evidence on the matter of the antrum size and its relation to bone mineral density (BMD).

The aim of this study was to compare the effect of AP-SG and AR-SG on the body composition, glycemic control and BMD in C57BL/6J Mice with obesity and type 2 diabetes.

\section{Materials and Methods}

\section{Animals}

All animal experiments were approved by the Animal Care and Use Committee of Nanjing Drum Tower Hospital, Nanjing Medical University (permission number: 2021AE0033). We have complied with all relevant codes of ethics.

Male C57BL/6J mice (4-6 weeks old) were obtained from the animal core facility of Nanjing Medical University (Jiangsu, China). The mice were housed in specific pathogen-free units of the Animal Center at Nanjing Drum Tower Hospital (with a 12 hours day $/ 12$ hours night cycle, $23 \pm 1{ }^{\circ} \mathrm{C}, 60-70 \%$ humidity) and fed a $60 \%$ high-fat diet (HFD; D12492, Research Diets, Inc., USA). The mice were induced T2DM by a high-fat diet for 4 months. Random blood glucose levels were measured with a hand-held glucose meter (OneTouch UltraVue, New Jersey, USA). Mice with a random blood glucose level $>16.0 \mathrm{mmol} / \mathrm{L}$ in 3 consecutive days were considered to be diabetic. The diabetic mice were randomly assigned into sham group, AP-SG and AR-SG group ( $\mathrm{n}=8$ in each group). Body weight, food intake, and fasting glucose (FG) levels were measured preoperatively and postoperatively at the second, fourth, sixth and eighth week. Oral glucose tolerance test (OGTT) was performed preoperatively and at postoperative eighth week. Two-hour OGTT with gavage of $50 \%$ D-glucose solution $(2 \mathrm{~g} / \mathrm{kg})$ were performed in all groups and plasma glucose levels were measured at $0,15,30,60,90$ and 120 min after gavage by tail bleeding in conscious mice. Eight weeks after surgery, the body fat content and bone mineral density were measured by dual-energy X-ray absorptiometry (DEXA) (XR-36; Norland Medical Systems, Fort Atkinson, WI, USA). The visceral white adipose tissue (WAT) and femurs were harvested for subsequent experiments.

\section{Sleeve Gastrectomy Surgical Procedure}

Mice were fasted overnight and anesthetized with isoflurane ( $2 \%$ for induction, $1 \%$ for maintenance). Under sterile conditions, a single $2 \mathrm{~cm}$ epigastric laparotomy was performed. As shown in Figure 1A, a standard technique for SG was performed. The stomach was externalized. For sham group, a similar length incision was made in the anterior gastric wall and then sutured using a 6-0 absorbable thread. In the antrum preservation group, the resection line started $1 \mathrm{~cm}$ from the pyloric ring (yellow arrow). In the antrum resection group, the resection line started as close as possible near the gastroduodenal junction (green arrow). Seventy percent of the stomach was removed and the incision site was sutured with 7-0 absorbable thread using a running suture. The serosal layer was inverted and sutured for reinforce. Abdominal 


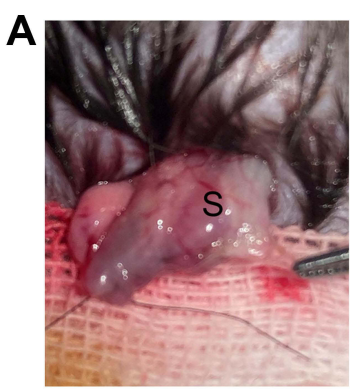

Sham

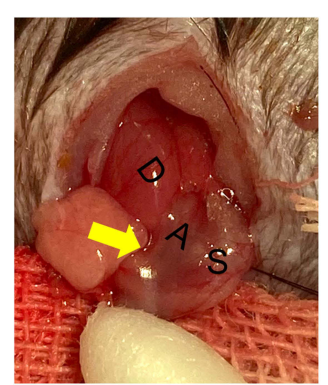

AP-SG

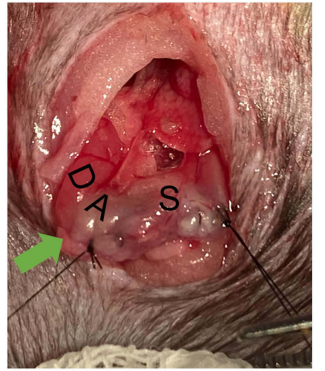

AR-SG
B

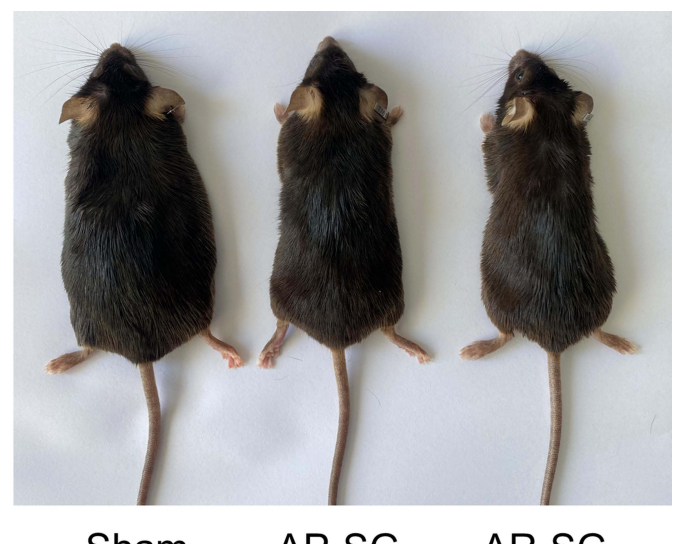

C

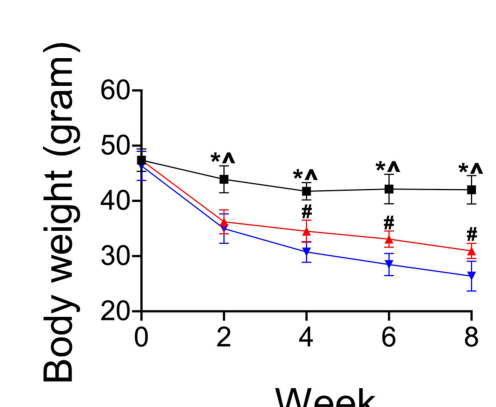

D

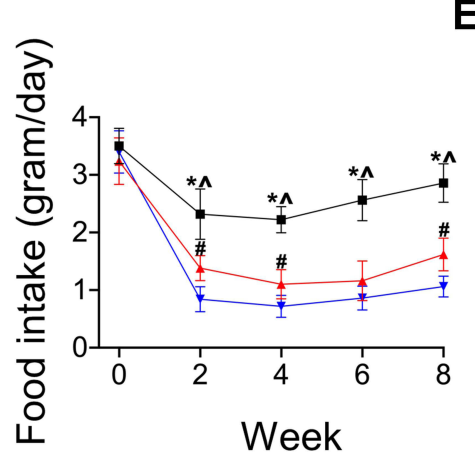

F

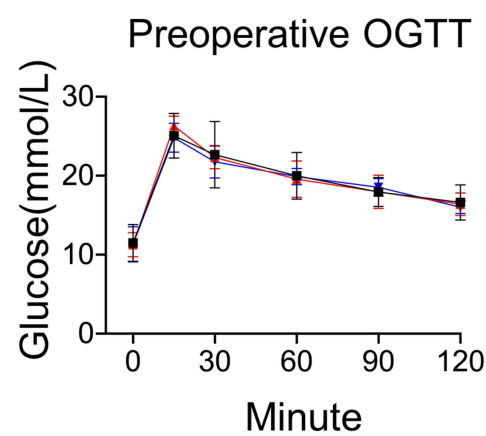

G

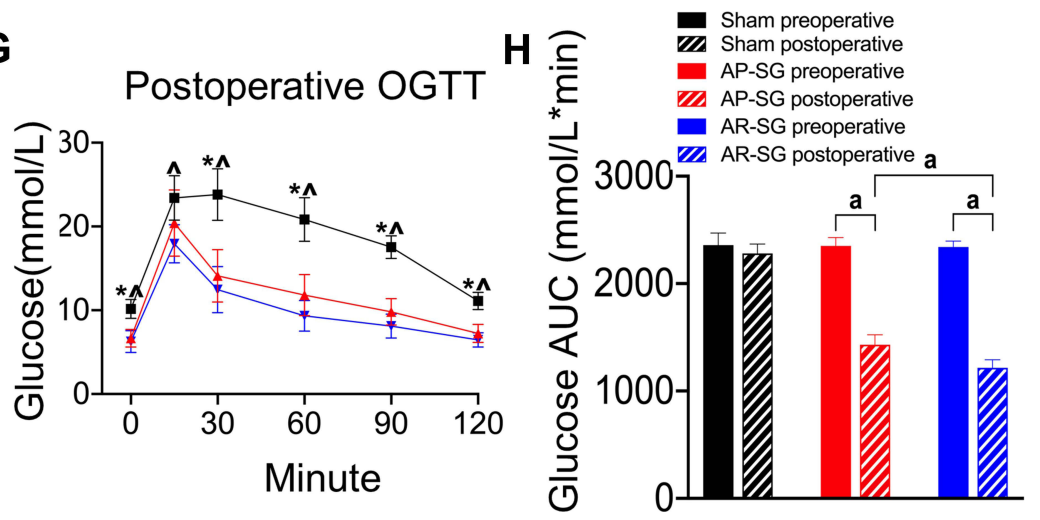

E

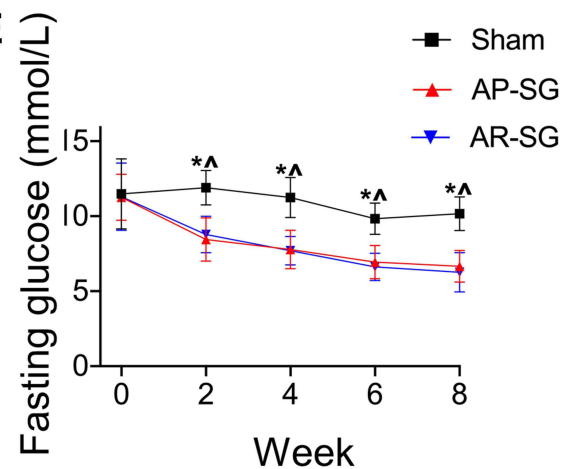

Figure I Sleeve gastrectomy (SG) alleviates obesity and glucose metabolic disorders in obese and T2D model. (A) Illustration of sham, antrum preservation SG (AP-SG) and antrum resection SG (AR-SG) operation. The resection line started I cm from the pyloric ring (yellow arrow). The resection line started as close as possible near the gastroduodenal junction (green arrow). D = duodenum; A = antrum; $\mathrm{S}=$ stomach. (B) Eight weeks after operation, representative appearances of mice in sham, AP-SG and AR-SG group are recorded. Illustrates changes in body weight (C), food intake (D), fasting glucose level (E) after operation. Oral glucose tolerance test (OGTT) results preoperative (F) and postoperatively $(\mathbf{G})$, and area under the curve (AUC) for glucose level $(\mathbf{H})$ are presented. Data $(n=8)$ are shown as mean \pm SD. $*$ Significant AP-SG compared with sham $(P<0.05)$. ^Significant AR-SG compared with sham $(P<0.05)$. ${ }^{\#}$ Significant AR-SG compared with AP-SG. ${ }^{a} P<0.05$.

closure was performed using 3-0 silk suture with a continuous suture technique. After allowing $24 \mathrm{~h}$ for the anastomoses to heal, the animals were kept on a liquid diet for 3 days before being returned to HFD for 8 more weeks. Body shapes was represented 8 weeks after operation in Figure 1B. The size and confirmation of antral resection were performed at the end of the study (Figure S1).

\section{Histological Analysis}

After mice were euthanized, their visceral WAT was fixed in $4 \%$ paraformaldehyde (PFA). The samples were then embedded in paraffin blocks and cut into 5- $\mu \mathrm{m}$ coronal slides by a microtome (Thermo Scientific, MA, USA). Tissue 
sections from sham, AP-SG and AR-SG group were stained by hematoxylin and eosin (H\&E) (Beyotime, Shanghai, China). Quantitative analysis of adipocyte size was performed using Image J (version 1.8.0).

\section{Micro-Computed Tomography (Micro-CT) Analysis}

After fixed in 4\% PFA, the microstructure and bone mineral density of mouse femurs were analyzed by a micro-CT scanner (mCT80; Scanco Medical AG, Bassersdorf, Switzerland) with $80-\mu \mathrm{m}$ voxel resolution. The images were then reconstructed to perform three-dimensional histomorphometric analysis with a Scanco Medical software. Mean/density (mg HA/ccm), bone volume $\left(\mathrm{BV}, \mathrm{mm}^{3}\right.$ ), bone volume per tissue volume (BV/TV, \%) were acquired by Scanco Medical software.

\section{Statistical Analyses}

SPSS software (version 26.0) and GraphPad Prism software (version 8.4) were used for the statistical analysis. One-way ANOVA analysis was used to compare surgical groups. The data were presented as mean values $\pm \mathrm{SD}$. $\mathrm{P}<0.05$ was considered statistically significant.

\section{Results}

\section{Body Weight, Food Intake and Glucose Level}

There were no significant differences in body weight and food intake among all groups preoperatively. Throughout the postoperative period, AP-SG and AR-SG showed significantly lower body weight and food intake compared with sham $(\mathrm{P}<0.05)$ (Figure 1C and D). Furthermore, AR-SG group had markedly reduced body weight and food intake compared to AP-SG group. There were no significant differences in FG and OGTT result among all groups preoperatively. Throughout the postoperative period, FG level declined significantly in AP-SG and AR-SG groups when compared with the preoperative level $(\mathrm{P}<0.05)$ (Figure 1E). At postoperative 8-week, FG levels declined significantly from preoperative level by $39.7 \pm 14.1 \%$ and $43.3 \pm 14.4 \%$ in AP-SG and AR-SG groups, respectively, but no significant difference was observed in FG between AP-SG and AR-SG group $(\mathrm{P}=0.598)$. Compared preoperatively (Figure $1 \mathrm{~F})$, postoperative AP-SG and AR-SG groups showed significant improvements in glycemic control $(\mathrm{P}<0.001)$ (Figure $1 \mathrm{G})$. However, AR-SG group showed significantly superior OGTT glucose AUC when compared with AP-SG and sham group ( $\mathrm{P}=0.004$ and $\mathrm{P}<0.001$, respectively) (Figure $1 \mathrm{H}$ ). Sham group preoperative and postoperative results did not differ significantly.

\section{Body Fat}

Similar to the changes in the glycemic control, AP-SG and AR-SG group had significantly lower body fat compared with sham group ( $\mathrm{P}=0.002$ and $\mathrm{P}<0.001$, respectively) (Figure $2 \mathrm{~A}$ and $\mathrm{B}$ ), and a significant reduction was observed in ARSG group than AP-SG group $(\mathrm{P}=0.017)$. Two months after operation, mice of all groups were euthanized. The visceral WAT is shown in Figure 2C, the adipose tissues of AR-SG group were smaller than sham and AP-SG group. Moreover, histology analyses of adipose tissue sections revealed that smaller adipocyte size in AR-SG group than sham and AP-SG $(\mathrm{P}<0.001$ and $\mathrm{P}=0.001$, respectively) (Figure 2D and $\mathrm{E})$.

\section{Bone Mineral Density}

Inspired by the encouraging effects of AP-SG and AR-SG on body fat, we further investigated their effects on BMD. Figure 3A and B illustrated that both AP-SG and AR-SG groups decreased total body BMD compared with sham group. Moreover, AR-SG induced greater BMD loss than AP-SG $(\mathrm{P}=0.023)$. Consistent with the total body BMD, AR-SG group showed significant osteoporosis bone loss in mouse distal femurs when compared with sham and AP-SG groups (Figure 3C). Quantitative analysis of bone parameters showed that the Mean density was significantly decreased in ARSG group compared with sham and AP-SG groups $(\mathrm{P}<0.001$ and $\mathrm{P}=0.028$, respectively) (Figure 3D). In addition, other parameters, such as BV and BV/TV also showed that AR-SG group lost more bone mass than sham and AP-SG groups (Figure 3E and F). 


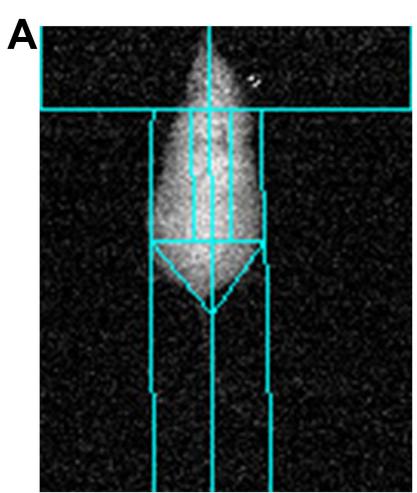

Sham

C

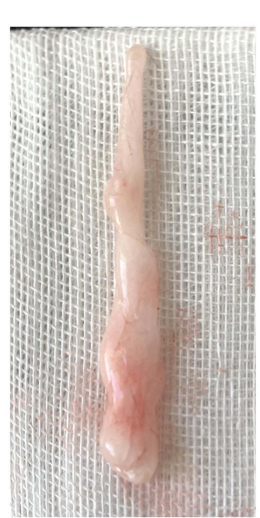

Sham

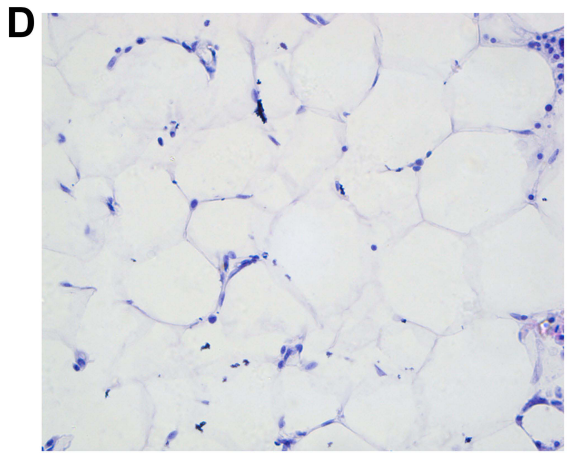

Sham

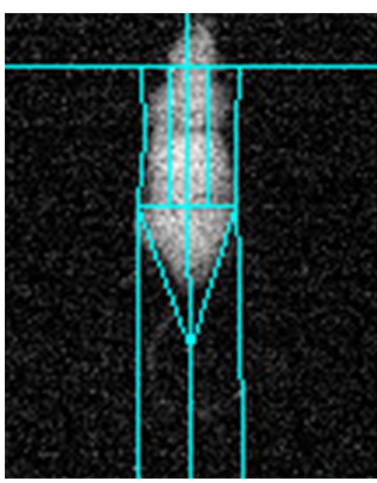

AP-SG

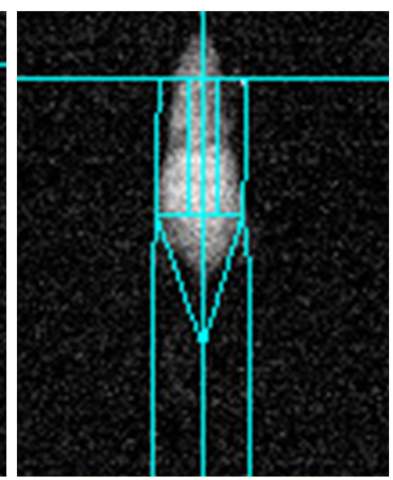

AR-SG

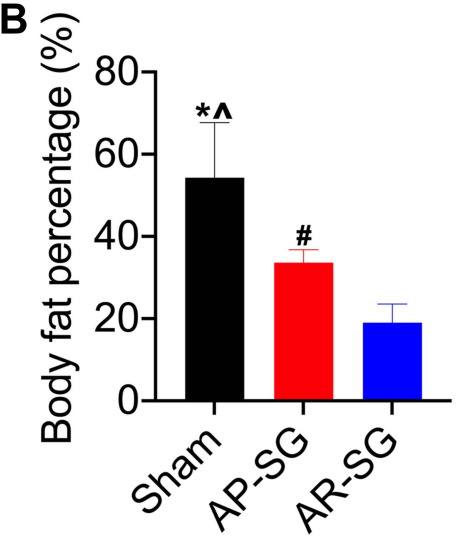

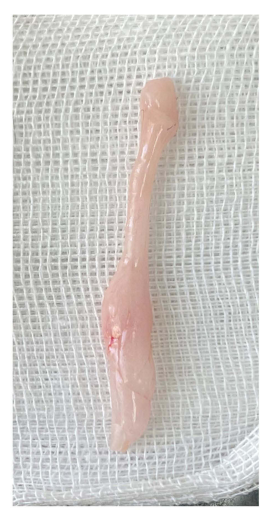

AP-SG

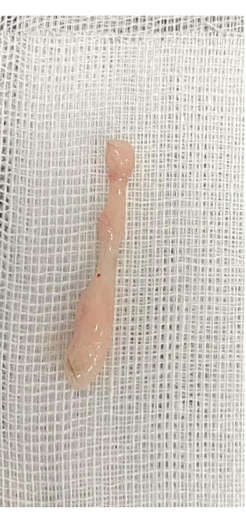

AR-SG

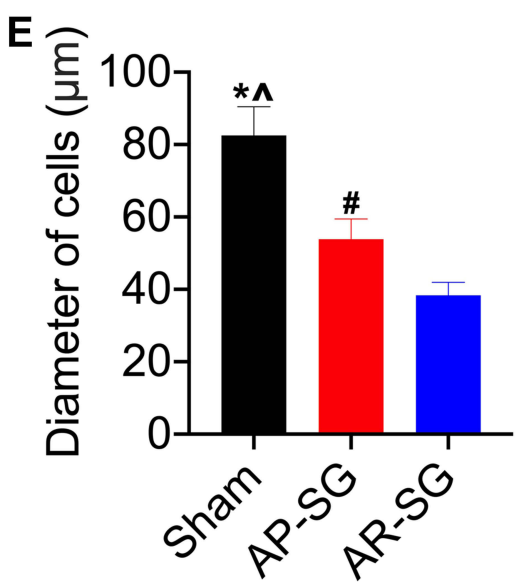

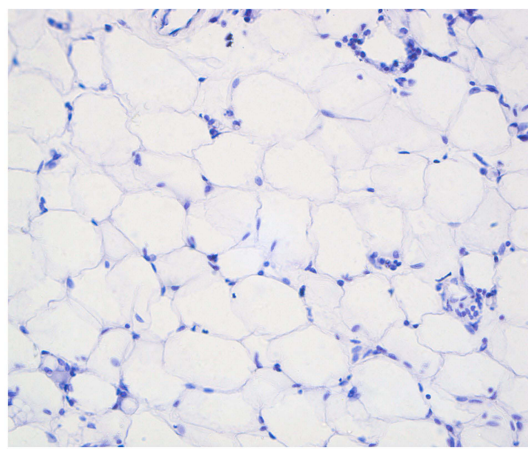

AP-SG

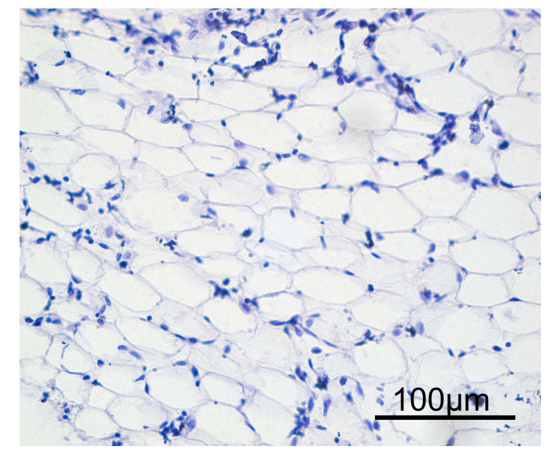

AR-SG

Figure 2 Antrum resection sleeve gastrectomy (AR-SG) induces significant body fat reduction than antrum preservation sleeve gastrectomy (AP-SG) in vivo. (A and B) Body fat content after AP-SG and AR-SG are measured by dual-energy X-ray absorptiometry. (C) Visceral adipose tissue after 8 weeks post-operation. (D) Hematoxylin and eosin images of visceral adipose tissue are visualized using an optical microscope. Scale bars: $100 \mu \mathrm{m}$. (E) Diameter of adipocytes of visceral adipose tissue in sham, AP-SG and AR-SG groups. *Significant AP-SG compared with sham $(P<0.05)$. ^Significant AR-SG compared with sham $(P<0.05)$. ${ }^{\#}$ Significant AR-SG compared with AP-SG.

\section{Discussion}

Nowadays, SG has been widely used as a stand-alone bariatric procedure. Numerous studies have documented significant weight loss and glucose improvement after SG. In this study, we demonstrated the changes in body weight, glycemic control and BMD after AP-SG and AR-SG. The major findings of the present study were that AR-SG achieved a lower percentage of body fat, a better glycemic control and a lower BMD compared to AP-SG.

The alteration in gastric motility induced by SG plays a role in the treatment of morbid obesity. After SG, the remaining gastric volume is considerably reduced and the gastric emptying reflex is modified. Both AP-SG and AR-SG can accelerate gastric emptying, and accelerated gastric emptying is able to enhance satiety signals, which leads to 


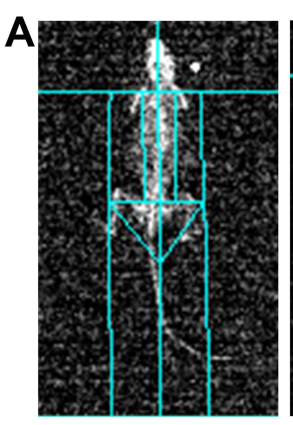

Sham

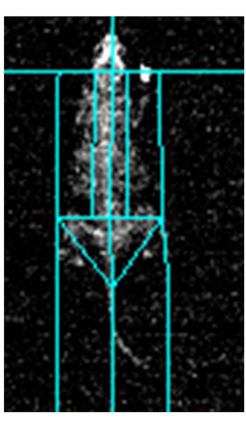

AP-SG

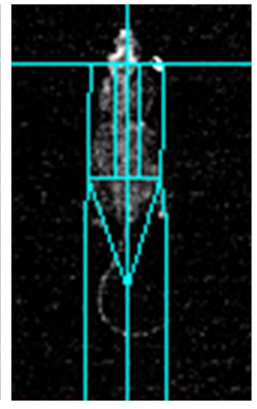

AR-SG
B
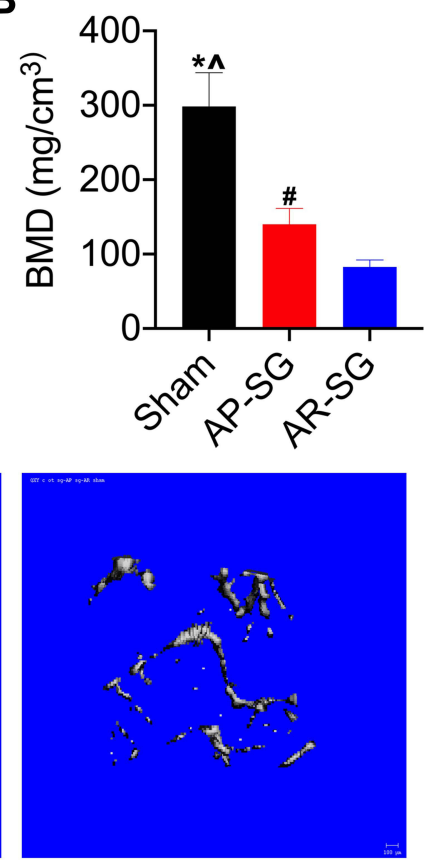

AR-SG

$\mathbf{F}$

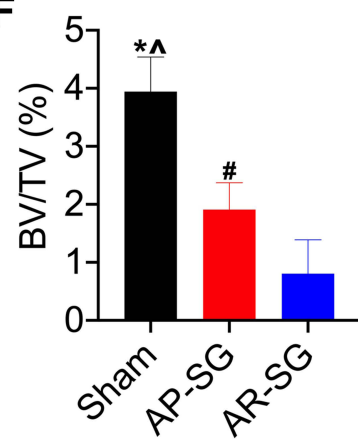

Figure 3 Antrum resection sleeve gastrectomy (AR-SG) causes significant bone mass reduction than antrum preservation sleeve gastrectomy (AP-SG). (A and B) Total body bone mineral density (BMD) after sham, AP-SG and AR-SG are measured by dual-energy X-ray absorptiometry. (C) Micro-computed tomography reconstruction images of cancellous bone in the distal femur. (D) Mean/density, (E) Bone volume (BV), and (F) Bone volume/tissue volume (BV/TV) in sham, AP-SG and AR-SG groups. *Significant AP-SG compared with sham (P<0.05). ^Significant AR-SG compared with sham $(P<0.05)$. " Significant AR-SG compared with AP-SG.

reduced food intake and body weight. ${ }^{21,22}$ Based on the results in this study, we assumed that AR-SG had a smaller volume and faster gastric emptying than AP-SG, which was indicated by a volumetric CT scanning. ${ }^{23}$ An important consequence of increased gastric emptying after SG is altered secretion of gastrointestinal hormones, in particular, GLP$1 .{ }^{24}$ Several studies have reported that increased gastric emptying contributes to glycemic control through upregulating GLP-1. ${ }^{23-25}$ Besides, Casella et al reported that improvement in insulin sensitivity correlated highly with both GLP-1 and body weight. ${ }^{25}$ Our findings go line with Nocca and Michalsky et al studies, ${ }^{8,26}$ where statistically significant improvement of body weight and glycemic control in AR-SG. Notably, for the AUC changes observed in AP-SG and AR-SGwhile statistically significant - the magnitude of difference between the two groups was modest. In this study, a GLP-1 and gastric emptying assessment should be conducted for further evaluation.

To date, detrimental effects on bone metabolism in relation to $\mathrm{SG}$ have been described. At a median per year after SG, Ieong et al observed a significant decrease in the femoral neck, femoral trochanter and total hip BMD following SG. ${ }^{27}$ In the meta-analysis of 22 studies by Jaruvongvanich et al, it focused only on SG and found significant decreases in total hip and femoral neck BMD and no significant change in lumbar spine BMD. ${ }^{28}$ But so far, no comparison of BMD has been 
reported between AP-SG and AR-SG. In this study, we not only evaluated total body BMD, but also assessed regional BMD in the distal femur. Consistent with the total body BMD, AR-SG group showed significant bone loss in distal femurs compared with AP-SG. Mechanisms involved are complex and multifactorial. The influence of mechanical unloading on reduced bone mass represents the simplest explanation. Our results showed that AR-SG exhibited significant weight loss than AP-SG. Due to a massive weight loss after SG, bone loss reflects a physiological adaptation to lower gravitational load demands. ${ }^{29}$ Another mechanism might be calcium and vitamin $\mathrm{D}$ deficiency due to malabsorption. In our study, food intake was markedly reduced in AR-SG, and we assumed that accelerated gastric emptying likewise resulted in partial malabsorption. In addition, Gkastaris et al perceived that massive fat mass reduction after SG may induce bone loss through changes in adipokines levels, but the specific mechanism is not well defined. ${ }^{30}$

The beginning of the gastric resection is still a controversial issue, and few studies tried to address the effect of antral resection on the outcome of SG. The findings of this study provide a certain reference to for clinical surgeons. According to our results, we found that weight loss and glycemic control were better in AR-SG. However, it should be noted that AR-SG seemed to develop more severe osteoporosis. The European Calcified Tissue Society has recommended that adequate daily calcium and vitamin D should be replenished after SG. ${ }^{31}$ This warrants serious consideration, especially for SG with shorter pyloric antrum area.

There are some limitations to address. First, the number of mice was small and postoperative observation was short, being only 8 weeks. Second, only the visceral WAT was used as a representative of white adipose tissue, more types of white adipose tissues are needed for further investigation. Last but not least, the animal model is not exactly the same as the clinical one. The small sample size could be interpreted as a pilot study. Further randomized clinical trials are indicated to confirm this finding.

\section{Conclusion}

Despite limitations of our study, we can conclude that both AP-SG and AR-SG can achieve a significant improvement in body weight and glycemic control, but AR-SG shows a lower percentage of body fat, a better glycemic control than APSG. We can also conclude that AR-SG is at higher risk of having lower bone mass compared with AP-SG. Therefore, more attention should be paid to BMD after SG, especially after AR-SG. A higher dose of calcium and vitamin D supplements should be considered in AR-SG postoperatively.

\section{Abbreviations}

SG, sleeve gastrectomy; AP-SG, antrum preservation sleeve gastrectomy; AR-SG, antrum resection sleeve gastrectomy; BMD, bone mineral density; FG, fasting glucose; OGTT, oral glucose tolerance test; HFD, high fat diet; DEXA, dualenergy X-ray absorptiometry; WAT, white adipose tissue; PFA, paraformaldehyde; H\&E, hematoxylin and eosin; BV, bone volume; BV/TV, bone volume per tissue volume.

\section{Ethics Approval}

All experiments with mice were authorized and performed in accordance with the Animal Care and Use Committee (permission number: 2021AE01033). All animal care and use protocols were approved by the Committee on the Ethics of Animal Experiments.

\section{Consent for Publication}

No conflict of interest exists in the submission of this manuscript, and all authors have approved the manuscript for submission.

\section{Acknowledgments}

We would like to thank Jiawei Li, Zhongyang Lv, Zhenhua Feng and Zhuang Qian for their kind advice of experimental skills. 


\section{Funding}

This study was supported by the Natural Science Foundation of Jiangsu Province (Grant No. BK20181155) and the 333 Project of Jiangsu Province (Grant No. BRA2017109).

\section{Disclosure}

The authors report no conflicts of interest in this work.

\section{References}

1. Debédat J, Amouyal C, Aron-Wisnewsky J, et al. Impact of bariatric surgery on type 2 diabetes: contribution of inflammation and gut microbiome? Semin Immunopathol. 2019;41:461-475.

2. Sjöström L, Narbro K, Sjöström CD, et al. Effects of bariatric surgery on mortality in Swedish obese subjects. N Engl J Med. 2007;357:741-752. doi:10.1056/NEJMoa066254

3. Puzziferri N, Roshek TB, Mayo HG, et al. Long-term follow-up after bariatric surgery: a systematic review. JAMA. 2014;312:934-942. doi:10.1001/jama.2014.10706

4. Schauer PR, Bhatt DL, Kirwan JP, et al. Bariatric surgery versus intensive medical therapy for diabetes - 5-year outcomes. $N$ Engl $J$ Med. 2017;376:641-651. doi:10.1056/NEJMoa1600869

5. Albaugh VL, Sharma G, Tu C, et al. Clinical significance of diabetes control before metabolic surgery. Surg Obes Relat Dis. 2021;17:1271-1278. doi:10.1016/j.soard.2021.03.031

6. Papailiou J, Albanopoulos K, Toutouzas KG, et al. Morbid obesity and sleeve gastrectomy: how does it work? Obes Surg. 2010;20:1448-1455. doi:10.1007/s11695-010-0148-5

7. Benaiges D, Más-Lorenzo A, Goday A, et al. Laparoscopic sleeve gastrectomy: more than a restrictive bariatric surgery procedure? World $J$ Gastroenterol. 2015;21:11804-11814. doi:10.3748/wjg.v21.i41.11804

8. Michalsky D, Dvorak P, Belacek J, et al. Radical resection of the pyloric antrum and its effect on gastric emptying after sleeve gastrectomy. Obes Surg. 2013;23:567-573. doi:10.1007/s11695-012-0850-6

9. Abdallah E, El Nakeeb A, Youssef T, et al. Impact of extent of antral resection on surgical outcomes of sleeve gastrectomy for morbid obesity (a prospective randomized study). Obes Surg. 2014;24:1587-1594.

10. Obeidat F, Shanti H, Mismar A, et al. The magnitude of antral resection in laparoscopic sleeve gastrectomy and its relationship to excess weight loss. Obes Surg. 2015;25:1928-1932. doi:10.1007/s11695-015-1642-6

11. McGlone ER, Gupta AK, Reddy M, et al. Antral resection versus antral preservation during laparoscopic sleeve gastrectomy for severe obesity: systematic review and meta-analysis. Surg Obes Relat Dis. 2018;14:857-864. doi:10.1016/j.soard.2018.02.021

12. ElGeidie A, ElHemaly M, Hamdy E, et al. The effect of residual gastric antrum size on the outcome of laparoscopic sleeve gastrectomy: a prospective randomized trial. Surg Obes Relat Dis. 2015;11:997-1003. doi:10.1016/j.soard.2014.12.025

13. Hussein AH, Khaled I, Faisal M. The role of the surgical resection distance from the pylorus after laparoscopic sleeve gastrectomy: a prospective cohort study from an academic medical center in Egypt. Patient Saf Surg. 2020;14:42.

14. Garay M, Balagué C, Rodríguez-Otero C, et al. Influence of antrum size on gastric emptying and weight-loss outcomes after laparoscopic sleeve gastrectomy (preliminary analysis of a randomized trial). Surg Endosc. 2018;32:2739-2745. doi:10.1007/s00464-017-5972-4

15. Shehata MA, Elhaddad A, El-Attar AA, et al. The effect of antrum size on weight loss, glucagon-like peptide-1 (GLP-1) levels, and glycemic control following laparoscopic sleeve gastrectomy in adolescents with obesity and type 2 diabetes. Obes Surg. 2021;31:4376-4385. doi:10.1007/ s11695-021-05590-9

16. Rousseau C, Jean S, Gamache P, et al. Change in fracture risk and fracture pattern after bariatric surgery: nested case-control study. $B M J .2016 ; 354$ : i3794. doi:10.1136/bmj.i3794

17. Pluskiewicz W, Bužga M, Holéczy P, et al. Bone mineral changes in spine and proximal femur in individual obese women after laparoscopic sleeve gastrectomy: a short-term study. Obes Surg. 2012;22:1068-1076. doi:10.1007/s11695-012-0654-8

18. Misra M, Singhal V, Carmine B, et al. Bone outcomes following sleeve gastrectomy in adolescents and young adults with obesity versus nonsurgical controls. Bone. 2020;134:115290.

19. Nogués X, Goday A, Peña MJ, et al. Bone mass loss after sleeve gastrectomy: a prospective comparative study with gastric bypass. Cir Esp. 2010;88:103-109. doi:10.1016/j.ciresp.2010.04.008

20. Stein EM, Silverberg SJ. Bone loss after bariatric surgery: causes, consequences, and management. Lancet Diabetes Endocrinol. $2014 ; 2: 165-174$. doi:10.1016/S2213-8587(13)70183-9

21. Melissas J, Koukouraki S, Askoxylakis J, et al. Sleeve gastrectomy: a restrictive procedure? Obes Surg. 2007;17:57-62. doi:10.1007/s11695-0079006-5

22. Melissas J, Daskalakis M, Koukouraki S, et al. Sleeve gastrectomy-a "food limiting" operation. Obes Surg. 2008;18:1251-1256. doi:10.1007/ s11695-008-9634-4

23. Vives M, Molina A, Danús M, et al. Analysis of gastric physiology after laparoscopic sleeve gastrectomy (LSG) with or without antral preservation in relation to metabolic response: a randomised study. Obes Surg. 2017;27:2836-2844. doi:10.1007/s11695-017-2700-z

24. Sista F, Abruzzese V, Clementi M, et al. The effect of sleeve gastrectomy on GLP-1 secretion and gastric emptying: a prospective study. Surg Obes Relat Dis. 2017;13:7-14. doi:10.1016/j.soard.2016.08.004

25. Casella G, Soricelli E, Castagneto-Gissey L, et al. Changes in insulin sensitivity and secretion after sleeve gastrectomy. Br J Surg. 2016;103:242248. doi:10.1002/bjs.10039

26. Nocca D, Picot MC, Donici I, et al. Evaluating the impact of the antrum size following laparoscopic sleeve gastrectomy: a randomized multicenter study. Surg Obes Relat Dis. 2020;16:1731-1736. doi:10.1016/j.soard.2020.06.041 
27. Ieong K, Ardila-Gatas J, Yang J, et al. Bone mineral density changes after bariatric surgery. Surg Endosc. 2021;35:4763-4770. doi:10.1007/s00464020-07953-2

28. Jaruvongvanich V, Vantanasiri K, Upala S, et al. Changes in bone mineral density and bone metabolism after sleeve gastrectomy: a systematic review and meta-analysis. Surg Obes Relat Dis. 2019;15:1252-1260. doi:10.1016/j.soard.2019.06.006

29. Diniz-Sousa F, Veras L, Boppre G, et al. The effect of bariatric surgery on gravitational loading and its impact on bone mass. Bone. 2021;153:116153. doi:10.1016/j.bone.2021.116153

30. Gkastaris K, Goulis DG, Potoupnis M, et al. Obesity, osteoporosis and bone metabolism. J Musculoskelet Neuronal Interact. 2020;20:372-381.

31. Julien P, Elena T, Christian M, et al. Bariatric surgery and skeletal health: a narrative review and position statement for management by the European Calcified Tissue Society (ECTS). Bone. 2021;22(154):116236.

Diabetes, Metabolic Syndrome and Obesity: Targets and Therapy

\section{Publish your work in this journal}

Diabetes, Metabolic Syndrome and Obesity: Targets and Therapy is an international, peer-reviewed open-access journal committed to the rapid publication of the latest laboratory and clinical findings in the fields of diabetes, metabolic syndrome and obesity research. Original research, review, case reports, hypothesis formation, expert opinion and commentaries are all considered for publication. The manuscript management system is completely online and includes a very quick and fair peer-review system, which is all easy to use. Visit http://www.dovepress. com/testimonials.php to read real quotes from published authors.

Submit your manuscript here: https://www.dovepress.com/diabetes-metabolic-syndrome-and-obesity-targets-and-therapy-journal 